\title{
Methods for characterizing, applying, and teaching CRISPR-Cas systems
}

\author{
Chase L. Beisel ${ }^{1,2,3}$ \\ ${ }^{1}$ Department of Chemical and Biomolecular Engineering \\ North Carolina State University, Raleigh, NC 27695, United States \\ ${ }^{2}$ Helmholtz Institute for RNA-based Infection Research (HIRI), \\ Helmholtz-Centre for Infection Research (HZI), 97080 Würzburg, Germany \\ ${ }^{3}$ Medical Faculty \\ University of Würzburg, 97080 Würzburg, Germany
}

Correspondence to Chase.Beisel@helmholtz-hiri.de 
1 Bacteria and archaea possess fascinating defense systems comprised of Clustered

2 Regularly Interspaced Short Palindromic Repeats (CRISPR) arrays and CRISPR-associated

3 (Cas) proteins whose characterization is driving a rapid and widespread technological

4 revolution. In their natural form, CRISPR-Cas systems confer adaptive immunity against

5 mobile genetic elements through a rich diversity of mechanisms and functions. This

6 functional diversity and the role of easy-to-design guide RNAs (gRNAs) led to the facile

7 translation of these systems into versatile tools impacting applications ranging from genome

8 editing and gene regulation to diagnostics, antimicrobials, and imaging agents. Ongoing

9 progress in applications such as human gene therapy and gene drives in turn has spurred

10 heated ethical debates, underscoring an urgent need to inform the wider population about

11 these technologies and their ramifications.

12 This special issue in Methods comprises two broad reviews and seven original

13 methods representing a broad sampling of current methodologies. Below is a brief overview

14 of each article grouped based on their primary relevance to the fundamental characterization

15 of CRISPR-Cas immune systems, implementation of CRISPR technologies specifically in

16 bacteria, and teaching CRISPR through hands-on modules intended for the classroom.

18 Methods for characterizing the biology of CRISPR-Cas systems. Five articles in the 19 special issue entail methodologies for identifying and characterizing the biomolecular 20 properties of CRISPR-Cas systems and their related components. Alkhnbashi and 21 coworkers provide a comprehensive review of available bioinformatics tools [ref1]. The 22 review principally describes tools for identifying and annotating CRISPR-Cas immune 23 systems, including finding CRISPR arrays and cas genes within genomic sequences, 24 properly orienting the arrays, identifying targets of the encoded CRISPR RNAs (crRNAs, the 25 natural form of gRNAs), and classifying the type and sub-type of each system. Furthermore, 26 available design tools for gRNAs relevant to CRISPR technologies are also described. The 27 bioinformatics tools for identifying CRISPR-Cas systems are particularly useful given the 
28 ubiquity of these systems in prokaryotes and their extensive diversity that is still being 29 understood and mined for technologies.

In the second review, Behler and Hess describe common approaches for interrogating

31 the mechanisms underlying crRNA biogenesis, with resulting insights across CRISPR-Cas

32 types and subtypes [ref2]. Prior work has shown that these mechanisms can vary widely,

33 requiring a broad set of techniques to pinpoint each mechanism and the responsible genetic

34 factors. The authors describe how these techniques helped reveal the different ways in

35 which a transcribed CRISPR array can be converted into individual crRNAs and aspects of 36 crRNA biogenesis that remain to be explored.

37 The first of the methods articles transitions the special issue from crRNA biogenesis to 38 target cleavage by the CRISPR effector nuclease responsible for immune defense. 39 Creutzburg and coworkers report a medium-throughput method to evaluate DNA cleavage 40 activity in vitro by the Type V-A nuclease Cas12a [ref3]. This single-effector nuclease offers 41 unique advantages over canonical Cas9 nucleases, such as staggered DNA cleavage or the 42 ability to generate crRNAs without accessory factors. As part of the method, the authors 43 targeted linear DNA with biotin on one end and a fluorophore on the other end, allowing the 44 facile measurement of the solution's fluorescence following the addition and removal of 45 streptavidin beads. The method was used to detect nuclease concentration and cleavage 46 rates, although it could be further applied to elucidate potential protospacer-adjacent motifs 47 (PAMs) or the impact of guide-target mismatches.

48 The next method from Gramelspacher and coworkers addresses a unique feature of 49 some Cas9 nucleases: the capacity to target RNA [ref4]. The authors detail how to 50 characterize RNA cleavage activity under in vitro conditions, with a focus on evaluating the 51 extent of cleavage, mapping the cleavage site, and determining whether the cleaved target 52 remains bound to Cas9. They also describe the purification of an anti-CRISPR protein (Acr) 53 and testing its ability to inhibit RNA cleavage by Cas9 in vitro. The method should be useful 54 when evaluating the extent to which other Cas9 nucleases exhibit this behavior as well as 55 the impact of different Acrs. 
The final method devoted to CRISPR biology takes a unique approach to the

57 characterization of Acrs. Acrs are thought to exist against virtually every CRISPR-Cas

58 system, yet Acrs have only been associated to-date with a small fraction of all CRISR-Cas

59 subtypes. Wandera and coworkers report the use of an E. coli-based transcription-

60 translation system (TXTL) to rapidly and scalably characterize different Acrs, using Acrs

61 against Cas9 as a case study [ref5]. In particular, they show how to modify a previously

62 published assay to minimize non-specific inhibition of gene expression associated with some

63 Acrs.

64

65 Methods for implementing CRISPR technologies in bacteria and archaea. The special

66 issue then turns from CRISPR biology to CRISPR technologies-specifically, programmable

67 gene editing and repression in prokaryotes [ref6]. Diallo and coworkers developed an

68 inducible two-plasmid system for genome editing with Cas9 for industrial strains of the

69 bacterial genus Clostridia. One of the plasmids encoded the recombineering template,

70 allowing for repair in coordination with Cas9 cleavage. The authors used this strategy to

71 delete a sporulation gene or insert a fungal cellobiohydrolase gene in one strain of

72 Clostridium beijerinckii followed by phenotypic characterization. The strategy was further

73 applied in another strain of $C$. beijerinckii to delete a gene conferring thiamphenicol

74 resistance and remove an endogenous plasmid, together allowing greatly improved

75 transformation of a plasmid selected with thiamphenicol.

Next, Depardieu and Bikard detail how to achieve programmable gene repression

77 with a catalytically-dead Cas9 (dCas9) in the bacteria Escherichia coli and Staphylococcus

78 aureus [ref7]. This mode of gene repression has been extremely effective for single or

79 multiplexed gene knock-down and has been easier to implement across bacterial species

80 than genome editing. Beyond showing how to generate and apply the basic constructs, the

81 authors incorporated origins-of-replication with broad host ranges. They also devised a

82 clever high-throughput screen to determine the optimal expression level of dCas9 
expression that maintains silencing activity without inducing a cytotoxic "bad seed" effect

84 observed previously by the authors.

Finally, Albers and coworkers describe an entirely distinct approach for achieving

86 CRISPR-based gene repression in archaea [ref8]. Rather than relying on dCas9 or another

87 heterologous nuclease, the authors co-opted the CRISPR-Cas system endogenous to the

88 archaeon Haloferax volcanii. The system was devoid of the nuclease-encoding subunit Cas3

89 as well as the processing enzyme Cas6; as a result, expressing a mature version of a

90 designed crRNA allowed the authors to achieve targeted gene silencing without competition

91 by endogenous crRNAs. Through this work, the authors provided basic design rules for

92 efficient gene silencing and demonstrated silencing of chromosomal and plasmid-encoded

93 genes, providing a strong foundation for CRISPR-based repression in other archaea.

94

95 Methods for using CRISPR in the classroom. The final article of the special entails how to

96 communicate the basic properties of genome editing with CRISPR technologies in a

97 classroom setting. Here, Ziegler and Nellen report a set of experiments to illustrate DNA

98 cleavage in E. coli [ref9]. They provide detailed protocols and instructions as well as lecture

99 materials, and the entire module can be completed in a day using simple and low-cost

100 materials and equipment. They also provided one round of student assessments indicating

101 positive reception as well as feedback to further improve the module.

102

103 Collectively, this special issue covers available methods for characterizing the rich diversity 104 of CRISPR biology, implementing a cross-section of current CRISPR technologies, and 105 porting CRISPR into the classroom. I hope you enjoy this issue and find it useful for your 106 own interests and pursuits.

107

\section{References}

109 1. Voss [review] - Bioinformatics tools

110 2. Hess [review] - Characterizing crRNA biogenesis and resulting insights 
3. van der Oost [method] - Characterizing cleavage by Cas12a in vitro

112 4. Zhang [method] - Characterization of RNA targeting by Cas9

113 5. Beisel [method] - Acr characterization using TXTL

114 6. Diallo [method] - Inducible editing with Cas9 in Clostridia

115 7. Bikard [method] - dCas9 in Strep, E. coli

116

8. Marchfelder [method] - CRISPRi in archaea

117

9. Ziegler [method] - CRISPR module for the classroom 